\title{
La intervención pedagógica como un reto de la formación universitaria: Hacia una práctica profesional articulada
}

\author{
Pedagogical Intervention as a Challenge of University Education. \\ Towards an Articulated Practice
}

\author{
Erick José de Padua Quesada Vargas' \\ División de Educación Básica, Centro de Investigación y Docencia en Educación \\ Universidad Nacional \\ Heredia, Costa Rica \\ erickdepadua07@gmail.com
}

Recibido 21 de marzo de 2013 • Corregido 28 de abril de 2013 • Aceptado 29 de abril de 2013

\begin{abstract}
Resumen. A continuación se aborda la importancia que tiene el proceso de intervención pedagógica que realizan los estudiantes y las estudiantes de la carrera de educación. Este proceso se puede considerar como médula espinal de la confrontación dialógica entre la teoría y la práctica. El principal objetivo es generar un análisis para reflexionar acerca de las verdaderas intencionalidades gestadas no solo desde cada curso universitario sino, además, valorar la creación de una propuesta articulada que se vea enriquecida e interrelacionada desde cada nivel académico. La principal intencionalidad es enriquecer la formación universitaria partiendo del planteamiento de interrogantes que permitan valorar de qué manera se articula el proceso, no solo desde la coordinación entre los cursos de cada periodo, sino también en la relación que debe existir en la construcción de un mayor dominio conceptual, práctico y actitudinal que se vaya gestando de manera graduada y secuencial. Se busca construir una alternativa de desarrollo que parta de la coordinación estrechamente secuenciada y enlazada de los procesos formativos.
\end{abstract}

Palabras claves. Pedagogía, práctica profesional, intervención pedagógica, formación universitaria, praxis, plan de estudios, malla curricular, habilidades, destrezas, necesidades educativas especiales, currículo universitario.

1 Magíster en Educación con énfasis en Docencia Universitaria (2004), bachiller y licenciado en educación con énfasis en Educación Rural I y II ciclos (2003), licenciado en Administración Educativa (2006), todos títulos otorgados por la Universidad Nacional. Actualmente académico propietario de la División de Educación Básica del Centro de Investigación y Docencia en Educación de la Universidad Nacional. Además, desde el 2005 profesor de cursos del Plan 200 del Ministerio de Educación Pública-Instituto de Desarrollo Profesional Uladislado Gámez. Experiencia profesional de 16 años como docente de I y ll ciclos y director de instituciones de educación primaria, tanto en instituciones de carácter público como privado. 
URL: http://www.una.ac.cr/educare

CORREO: educare@una.cr

\begin{abstract}
The following addresses the importance of the pedagogical intervention process undertaken by the students of the Faculty of Education. This process can be considered as backbone of dialogic confrontation between theory and practice. The main objective is to generate an analysis to reflect on the true intentions that are born not only from each university course, but also to evaluate the creation of a coordinated and interrelated proposal enriched by each grade level. The main intention is to enrich university education starting from posing questions that allow us to assess how the process is structured, not only from the coordination achieved between courses each period, but also from the relationship that should exist in the construction of conceptual, practical and attitudinal strengths that are created both gradually and sequentially. The construction of an alternative development proposal is seeked; one which starts with the learning processes' sequenced and linked coordination.
\end{abstract}

Keywords. Pedagogy, professional practice, educational intervention, university training, curriculum, special education needs, university curriculum.

Sin lugar a dudas, el desarrollo de un profesional en educación implica una serie de retos amplios y profundos a lo largo de su formación universitaria. En este sentido, son diversas las variables que interactúan entre sí para lograr espacios significativos que le permitan la construcción de un modelo docente adecuado a las diferentes realidades educativas a las cuales se enfrentará a lo largo de su labor. En este sentido, la formación universitaria procura abarcar tres grandes dimensiones: la investigación, la docencia y la extensión, todas ellas canalizadas y desarrolladas a lo largo de dicha formación. Estas tres dimensiones tienen como objetivo primordial generar un diálogo directo y estrecho entre los elementos teóricos, y los ejercicios prácticos a los cuales cada estudiante es confrontado desde los cursos universitarios que recibe durante su vida estudiantil.

La práctica profesional es conceptualizada por muchos como la intervención pedagógica a la que se exponen los estudiantes y las estudiantes y resulta ser fundamental en su formación, ya que como se encuentra establecido en la malla curricular de las carreras de la División de Educación Básica (DEB), del Centro de Investigación y Docencia en Educación (CIDE), de la Universidad Nacional de Costa Rica (UNA), los cursos están distribuidos de tal manera que logren desarrollar un equilibrio que permita al estudiantado, además de las horas teoría recibidas en las aulas universitarias, también horas de estudio independiente y así mismo horas de carácter práctico. Tomando en consideración lo anterior, se podría pensar que con esta integración se debe fortalecer la "praxis" en el estudiantado universitario, una praxis orientada y enriquecida por el profesorado universitario a cargo de los respectivos cursos.

El profesorado universitario debe generar un análisis profundo que le permita aprovechar al máximo las diferentes situaciones de aprendizaje que se promuevan o establezcan durante el desarrollo de un curso universitario, no visto el curso como una isla separada de un continente formativo, sino relacionado con el resto de la malla curricular para generar un enlace que sirva como base para cursos posteriores y de ese modo lograr el desarrollo de diferentes habilidades cognoscitivas, socio-afectivas y psicomotrices. Ahora bien, se corre el riesgo de no lograr siempre esta articulación y, si se carece de espacios en que se reflexione al respecto, podría existir una incoherencia a lo largo de la formación. 
Ante esto, la intervención pedagógica se convierte en la columna vertebral del quehacer universitario como principal gestor de aprendizajes significativos en su estudiantado en formación, ya que este proceso articula, de manera vivencial, todo aquello que el estudiantado logran construir desde las aulas universitarias y lo confrontan con la experiencia vivencial que se desarrolla en las aulas escolares, es decir, con aquellas situaciones cotidianas en las que se encuentra inmersa una institución en el diario quehacer educativo.

Este aspecto es un foco de atención primordial por parte de los docentes universitarios, ya que de no orientarse de una forma adecuada, podría resultar que sea más fuerte el modelo que reciben los estudiantes universitarios por parte de sus docentes anfitriones que la misma formación que pretende desarrollar la docencia universitaria. Este punto remite, automáticamente, a realizar una valoración profunda acerca del perfil y función que debe cumplir un docente anfitrión que reciba en sus aulas escolares a un estudiante universitario para que realice la aplicación y desarrollo de sus horas prácticas, de tal modo que se pueda garantizar una coherencia sustancial entre dicha vinculación, que iría desde lo que se analiza y profundiza en el aula universitaria y lo que sucede en la vida escolar diaria.

El punto anterior se convierte en un inmenso reto de la academia universitaria, ya que no solo debe aproximar al estudiante universitario a la dimensión cotidiana de su futura labor profesional, sino que también se convierte en un insumo directo para los profesores que imparten el curso, ya que constantemente deberían estar retomando aquellas situaciones que generen en el estudiante universitario preguntas, inquietudes, desafíos, situaciones de desequilibrio entre lo que se propone desarrollar y lo que pueden alcanzar realmente.

La metodología y los criterios de evaluación que se establezcan desde el programa de un curso determinado deben poseer básicamente tres grandes características: ser funcionales, coherentes y precisos en lo que se quiere lograr, no basta con que el estudiante "haga y haga", se busca en todo momento que lo que haga tenga significatividad, es decir, debe lograr en el estudiante universitario un objetivo definido y responder al perfil de salida de la carrera; en caso de que carezca de esta condición, se podría estar desarrollando un "activismo irrelevante".

Quienes desarrollan cursos universitarios podrían detenerse a pensar y a tratar de contestar preguntas básicas y necesarias como las siguientes: ¿Son realmente significativos para el estudiante universitario los trabajos que se le asignan desde el curso que imparto?, ¿cuáles serán las principales habilidades que lograrán desarrollar?, ¿en realidad se hace una evaluación integral de los alcances o limitaciones que representó para el estudiante la actividad propuesta?, ¿se retoman estos aspectos en las plenarias presenciales o en la revisión que hace el profesor?, ¿fue pertinente lo que se propuso y es válido de aplicación para este mismo curso si lo debo impartir en experiencias futuras?, ¿qué actividades o propuestas son necesarias de reformularse, retomarse o incorporar como nuevas? Una vez que los estudiantes y las estudiantes han realizado determinada actividad, ¿qué logrará realizar ahora y qué antes no podía por no tenerlo claro? Este tipo de razonamientos podría evitar el caer en prácticas rutinarias, las cuales si no se reflexiona sobre las mismas serían hasta inútiles. 
URL: http://www.una.ac.cr/educare

CORREO: educare@una.cr

Comprender la formación universitaria resulta un ejercicio amplio, complejo y cauteloso, ya que no solo se centra en las actividades pedagógicas que el estudiantado universitario desarrolla, sino contempla un sinnúmero de variables de carácter socio-culturales. Por ejemplo, una variable que siempre debe estar presente es la valoración del grado de madurez académica con que cuentan los estudiantes y las estudiantes universitarios, no es solo posicionarse en una situación de rendimiento académico en el aula universitaria, sino comprender cómo se enfrenta el estudiante en otros espacios (como el aula en el momento en que asume un grupo escolar). Resulta vital contemplar si en realidad los estudiantes están preparados para asumir retos de carácter profesional, los cuales no solo implican un dominio meramente conceptual, sino también deben contemplar una plataforma intelectual que sirva como un andamiaje integral para la aplicación de respuestas pertinentes y atinadas.

Esta situación abre la puerta para valorar concienzudamente qué puede hacer un estudiante dependiendo del nivel universitario en que se encuentre. Del mismo modo, remite al personal docente universitario a replantearse qué le puede solicitar a un estudiante en su práctica de acuerdo con lo que la misma formación ha logrado desarrollarle. Si se parte de este punto, se podría determinar que algunos momentos no se encuentran bien ubicados o que los niveles de intencionalidad sobrepasan lo que el estudiantado puede lograr.

Esto sería visualizado como una incongruencia formativa, ya que al estudiante se le podría estar pidiendo que realice operaciones procedimentales que ni siquiera la misma formación universitaria ha logrado ofrecerle. Por ejemplo, solicitarle a un estudiante que asuma un grupo escolar en el cual se encuentren estudiantes a los cuales se les deben realizar adecuaciones curriculares significativas y que la misma formación específica al respecto no le haya sido brindada profundamente. Esta inquietud siempre ha sido un punto de reflexión personal, ya que si se analiza a la luz de la experiencia, quizás la estructura práctica y aplicativa de las intervenciones pedagógicas podrían no estar bien ubicadas u orientadas adecuadamente y las exigencias a que es sometido el estudiantado, podrían corresponder a un nivel formativo superior.

Este punto implicaría, entonces, por un lado, una serie de condiciones anómalas que afectarían el carácter educativo en los estudiantes y las estudiantes y, de paso, generar abismos secuenciales que implicarían una descoordinación entre los niveles formativos, tal y como lo indican diversos pedagogos.

A partir de mi experiencia como coordinador del curso "Construcción pedagógica desde el aula escolar" (División de Educación Básica, Centro de Investigación y Docencia en Educación, Universidad Nacional, s. f.), y luego de haber conocido los espacios o acercamientos que se le propician a los estudiantes y las estudiantes, se generaron en mi persona diferentes inquietudes; sin embargo, una de las que más a menudo recurría a mi mente se relacionaba con responder si la intervención pedagógica que realizan los estudiantes en el nivel de diplomado se encontraba debidamente ubicada o si, por el contrario, correspondería más bien a una intervención que deberían ejecutar los estudiantes en un nivel más avanzado. En ello consideraba como un factor determinante el que hubiesen alcanzado un mayor nivel de desarrollo personal que se viera 
reflejado en su vida universitaria. Esto no implica que desaparezca la intervención pedagógica en el nivel de diplomado, sino más bien reformular qué es lo que el estudiantado debe realizar en este nivel o bien valorar a qué es lo que puede enfrentarse como estudiante inicial en su formación universitaria.

Ante lo anterior, no cabe duda que el instrumental teórico-práctico de un nivel de diplomado es básico y, si bien determina en gran profundidad el perfil de un futuro docente, aún existe un largo recorrido por alcanzarse, ya que muchos estudiantes apenas comienzan a descubrir la magnitud y la importancia que asume un profesional de educación en el plano social. Al respecto, replantearse si un estudiante que cursa el nivel de diplomado está en realidad preparado para realizar en aulas escolares ejercicios prácticos docentes es un tema polémico y de mucha discusión académica.

Ahora bien, para lograr una adecuada intervención pedagógica, gestionada y direccionada desde los cursos universitarios, resulta necesario generar un enlace directo entre lo que establece el perfil de ingreso y el perfil de salida deseable para cada especialidad, todo ello, a partir de los objetivos propuestos en cada curso. En este sentido, el manejo y la claridad del personal docente universitario es fundamental, ya que este grupo es el garante de lo que los estudiantes pueden realizar y que esto sea una construcción pertinente, es decir, cuáles habilidades reales son las que han logrado alcanzar durante el desarrollo de cada curso y, posteriormente, que sean esas destrezas o habilidades las que eventualmente les sean evaluadas de manera integral, en donde no solo se valore el desempeño estudiantil, sino también la efectividad de toda la propuesta educativa como tal.

De acuerdo con ello, la coordinación entre los cursos universitarios, ya vista en un mismo ciclo universitario o entre diferentes niveles formativos, es vital para procurar un desarrollo coherente y lógico, el cual debe partir de las habilidades y destrezas que va alcanzando la formación universitaria de una manera procesual.

Para lograr dosificar y generar aprendizajes pertinentes, se debe partir fundamentalmente del perfil de ingreso del estudiantado al iniciar su carrera universitaria. Quizás este sea uno de los aspectos más importantes por valorar para determinar lo que puedan o no desarrollar en el nivel de diplomado. De acuerdo con lo expresado por algunos docentes que comparten con esta población, en este nivel formativo se percibe, en una parte representativa, un cierto rezago académico, pues apenas comienzan a establecer nuevos esquemas mentales y procedimentales. Por ejemplo, en el diplomado se pueden detectar serias deficiencias formativas, algunas evaluadas en las Pruebas Nacionales de Bachillerato: pésima ortografía, redacción confusa, baja expresión oral, poca oratoria, razonamiento y conocimiento lógico matemático confuso, lectura superficial de textos, incorrecta aplicación de signos de puntuación, baja abstracción de ideas, poco pensamiento crítico, carencia significativa en el hábito de lectura y otras, lo cual se convierte en todo un reto para los profesores universitarios, ya que además de retomar estas deficiencias, paralelamente se debe lograr la formación del perfil docente idóneo. Analizados desde una dimensión objetiva, estos aspectos pasan a ser una prioridad formativa para lograr 
URL: http://www.una.ac.cr/educare

CORREO: educare@una.cr

garantizar resultados integrales, que precisamente le permita, a esta población, enfrentarse de manera satisfactoria y más segura a la labor de aula.

Desde este perspectiva, resulta fundamental realizar algunas consideraciones sobre lo que implica atender un grupo escolar. El reto del estudiantado universitario al momento de enfrentarse a la realidad educativa y generar una mediación directa da como resultado un caleidoscopio vivencial, ya que asumir un grupo escolar implica la puesta en práctica de diferentes habilidades y destrezas profesionales, algunas de ellas de mucha atención y con un referente cognitivo, metodológico y actitudinal bien desarrollado.

El desarrollo de habilidades comunicativas es quizás la base primordial para que se gesten procesos educativos de calidad, partiendo de una adecuada impostación de la voz, en donde intervienen elementos fundamentales como: entonación, dicción correcta de las palabras, volumen y proyección de la voz, seguridad expositiva, ordenamiento de ideas, capacidad para aclarar dudas y para enfrentarse a imprevistos que impliquen un razonamiento adecuado, una capacidad de respuesta rápida y precisa.

Por otro lado, los estudiantes universitarios y las estudiantes universitarias deben contar con un abanico amplio de estrategias didácticas que fortalezcan su formación profesional. Este aspecto constituye un eje medular, por cuanto un docente de primaria debe desarrollar diferentes disciplinas científicas a partir de su mediación pedagógica.

Por lo tanto, esta formación debe ofrecerle una plataforma didáctica que le permita dominar ampliamente cada asignatura por impartir y facilitarle un abordaje metodológico diferenciado; esto es, no se debe masificar el desarrollo de estrategias de una manera indiscriminada, pues este procedimiento puede dar como resultado de que los aprendizajes carezcan de significatividad. Algo tan vital como cuestionarse si se debe mantener la ambientación de aula escolar, de la misma manera cuando se imparten lecciones de una asignatura en específico en contraste con otras asignaturas. Esto invitaría a la reflexión de cómo generar mejores espacios escolares para la construcción de aprendizajes. En este sentido, el estudiantado universitario debe valorar en qué circunstancias y de qué forma está compartiendo ideas, datos, inquietudes y sobre todo un diálogo interno que le aproxime al dominio que va alcanzando en su propia formación profesional. Resulta preciso retomar y aclarar aspectos tales como las características actitudinales de la población estudiantil, espacios educativos alternos dentro de la misma aula, la incorporación de recursos tecnológicos, reglas o límites establecidos, el tipo de liderazgo que se ejerce, entre otros aspectos.

Otro elemento que debe obtener un estudiante universitario en su formación académica es el dominio preciso del planeamiento didáctico. Esto implica una adecuada planificación de las lecciones, donde contemple diferentes aristas de la epistemología, genere una adecuada relación horizontal y vertical en el planeamiento como un todo, proponga una línea secuencial en la cual cada niño y cada niña logre alcanzar el desarrollo de estructuras cognitivas a partir de su estilo y ritmo de aprendizaje y, paralelamente, desarrollar valores y actitudes que le permitan una aplicabilidad inmediata en su modo de vida. En este mismo punto, se podría considerar la 
profundidad de dominio y de análisis de la evaluación, la cual no se puede enmarcar en una simple representación numérica, sino que, por el contrario, se debe convertir en el reflejo de una experiencia en la cual no solo el estudiante sea evaluado, sino que además se contemplen diferentes aspectos que intervienen directa o indirectamente en dicho momento práctico, tales como variedad, calidad y efectividad de la actividades, pertinencia de los materiales utilizados, calidad de las explicaciones realizadas, grado de involucramiento o de apoyo por parte del núcleo familiar, condiciones ambientales del aula y de la misma institución educativa: nivel de temperatura imperante, ruido, hacinamiento, presencia de malos olores, ventilación, iluminación y otros.

Por otra parte, otro dominio cognoscitivo que deben desarrollar los estudiantes y las estudiantes universitarios es el conocer los principales postulados pedagógicos subyacentes en la labor educativa a partir de diferentes autores, ya que al ser la pedagogía la ciencia encargada del estudio profundo y específico de la educación, deben poseer puntos de comparación al respecto, por ejemplo, conocer los postulados de autores diferentes e incluso contrapuestos en sus planteamientos.

Otro aspecto es el manejo y dominio de información relacionada con la psicología humana. Básicamente en el desarrollo de aprendizajes es fundamental un dominio que permita una atención científica a la población estudiantil con la cual se trabajará. Al respecto, se debe recurrir nuevamente al conocimiento y valoración de postulados tan importantes como los establecidos por diferentes teóricos.

Quizás un elemento fundamental que podría pasar desapercibido es el dominio conceptual y científico de los diferentes tópicos a desarrollar en el aula escolar. Esto corresponde necesariamente a la capacidad de poder ampliar de manera precisa y amplia todos aquellos contenidos que se encuentran establecidos en los Programas de Estudio del Ministerio de Educación Pública (MEP, 2005) en las diferentes asignaturas por impartirse. Como es bien sabido, este aspecto implica que las estudiantes y los estudiantes universitarios logren desarrollar un enriquecimiento cultural amplio, pues no solo deberían poseer nociones básicas o elementales sino conocimientos amplios, diversificados y paralelos que les permitan dar explicaciones enriquecidas para satisfacer la sed de conocimiento y curiosidad del estudiantado en edad escolar. Al respecto, esto no implica que se conviertan en "sabelotodos", sino que sean capaces de generar en sus estudiantes inquietudes que les permitan y propicien actitudes investigativas, aspecto valioso que el mismo docente desarrolla durante el ejercicio de sus funciones.

Ser docente implica, entre muchas otras cosas, ser un conocedor del referente histórico social de la humanidad, con un bagaje cultural amplio que le permita generar relaciones sociopolíticas que le faciliten la comprensión de cómo las estructuras políticas, ideológicas, religiosas y culturales influyen de manera significativa en la construcción social existente; valorar, así, aspectos apropiados e inapropiados que han dado como resultado el momento histórico actual, para lo cual necesita un grado de objetividad que le facilite una lectura crítica de la realidad contemporánea. 
URL: http://www.una.ac.cr/educare

CORREO: educare@una.cr

Otro reto que surge en la formación académica es el desarrollo del pensamiento crítico. Probablemente para muchas personas esta es una condición o destreza sencilla de desarrollar; pero, sin lugar a dudas, implica todo un cuestionamiento y razonamiento profundo, ya que en la mayoría de los casos consiste en estructurar y revisar las propias creencias, paradigmas y cosmovisiones interiores, las cuales la mayoría de veces han implicado años de construcción, situación que mencionan diferentes teóricos. Este reto representa un análisis de la intrínseca estructura mental, la confrontación de los propios esquemas cognitivos, los cuales podrían estar sustentados en una escala de valores y actitudes que trascienden desde los primeros años de infancia. Estos adquieren un elevado nivel de protagonismo en la personalidad, se ven reflejados en la manera de ver el mundo y, de hecho, a la hora de educar para la trasformación de este. Este tipo de pensamiento es fundamental para comprender el comportamiento de estructuras políticas, económicas y socio-culturales, ya que se convierte en el principal agente de una educación subversiva que puede comprenderlas desde una perspectiva pro-activa.

Otra característica profesional que deben poseer los estudiantes y las estudiantes universitarios es la habilidad de generar estrategias amparadas en diferentes enfoques investigativos. Esta condición es, probablemente, otra de las destrezas más importantes que se debe desarrollar a lo largo de su vivencia universitaria, ya que su labor profesional girará en gran medida en procesos que requieran ser analizados y comprendidos desde una logística científica. La etnografía, precisamente, otorga, a los profesionales de la educación, un punto de partida amplio para conocer y reflexionar sobre el comportamiento de los diferentes grupos sociales, sus formas de ver el mundo y cómo dentro de todas estas culturas interactúan los símbolos y su respectiva simbología (interaccionismo simbólico) y cómo esto determina procedimientos y construcciones mentales, las cuales definen el proceder de los sujetos sociales, todo ello, enlazado al momento histórico y situaciones predominantes.

Conocer a ciencia cierta el proceder de un grupo determinado resulta vital para entender el porqué de sus acciones, sin que necesariamente se justifiquen las mismas; valorar el porqué se brindan determinados resultados ante situaciones en las cuales quizás no se actuaría de la misma manera. Por ejemplo, en mi caso, no habría logrado comprender por qué, en una institución determinada, los estudiantes se llevaban para sus casas las llaves con que se abren los tubos (comúnmente llamadas mariposas) o las tapas de los sentaderos de los servicios sanitarios, hasta que no participé en el año 2000 del Censo Nacional, el cual me correspondió desarrollar en las "Gradas de Cristo Rey", al sur de la provincia de San José. En una de las viviendas visitadas me encontré con que no poseían servicio sanitario, sino que recogían el excremento en una cubeta de pintura de 5 galones, sobre la cual colocaban una tapa sanitaria. Luego de contener cierta cantidad de contenido fecal, era vaciada en las márgenes del Río María Aguilar. Quizás este sea un ejemplo duro y escalofriante, curiosamente, fue la respuesta exacta para comprender por qué se llevaban de la institución educativa objetos que no tenían en gran medida un valor económico, sino que poseían características de necesidad dentro de un carácter de escases en la subsistencia cotidiana, pues representaba una cierta adquisición de un artículo indispensable para mejorar su condición de vida paupérrima. 
La habilidad fundamental de sistematizar información es también un factor contundente, aquí subyacen diferentes aspectos de carácter organizativo y estético-funcional, ya que implica el desarrollo de una habilidad administrativa, documental y de archivo formal de la información. Esta habilidad les permita responder a los procedimientos administrativos-burocráticos que la estructura gubernamental les solicitará de manera bombardeada a lo largo de su función docente. Al respecto, es importante reflexionar y retomar la cantidad de horas que invierte un educador en el desarrollo de funciones completamente alejadas de su labor pedagógica, realizando informes que lejos de permitirle mayor calidad, se convierten en obstáculos directos que impiden la planificación didáctica y que, normalmente, resultan en procedimientos estadísticos que no sirven para nada más que eso, para generar estadísticas numéricas que terminan depositadas en informes de carácter neutro o transitorio, nunca para ahondar realmente en el mejoramiento de la calidad educativa. Se separa, claro está, el concepto de "educación" versus "información" de las personas, en donde hasta la información recibida podría cuestionarse dependiendo del grado o nivel en que se imparta y los temas seleccionados. Se podría cuestionar de qué forma contribuyen los contenidos o temas a desarrollar en la vida social inmediata de cada estudiante comprendido como actor social.

Otra de las habilidades necesarias en este estudiante universitario es el dominio de principios básicos de administración educativa, aspecto relevante para poder cumplir con requerimientos administrativos tales como: La fórmula 14, la cual es el sumario de las calificaciones finales obtenidas por los estudiantes y las estudiantes escolares luego de cada curso lectivo; el Plan operativo anual, que consiste en una planificación de los proyectos o procesos en que se verá inmersa la institución educativa, con el propósito de mejorar las condiciones de su dinámica administrativa y curricular; el Expediente acumulativo de las estudiantes y los estudiantes, el cual resulta un enriquecedor documento que recopila aquellos aspectos relevantes que han influenciado de manera positiva o negativa el rendimiento escolar; las Notas o calificaciones, que se envían al hogar como un informe de los avances de cada periodo, estadísticas de ingresos, egresos, traslados, estudiantes con sobre edad, repitencia, adecuaciones curriculares y otros. Quizás en este punto se centra el mayor distractor pedagógico de los sistemas educativos, los cuales en lugar de propiciar el desarrollo de condiciones profesionales que fortalezcan la dimensión didáctica y pedagógica, orientan de manera sistemática, desgastante y mal intencionada el desarrollo de actividades burocráticas, administrativas y poco relevantes. Todas, para poder justificar datos relacionados con repitencia y expulsión (la cual es cobijada con el nombre de deserción, de esa forma los estudiantes son los traidores o desertores del sistema educativo, el cual se mantiene sacro y benevolente).

Quienes han laborado en las aulas escolares, saben el gran desgaste que implica la dimensión administrativo-burocrática a que se enfrenta la labor docente y de cómo esto se convierte en un distractor propio de la labor educativa.

El manejo legal y procedimental de la aplicación de adecuaciones curriculares es otro aspecto clave, ya que adquiere una dimensión vital para garantizar el derecho y la necesidad de que personas con necesidades educativas especiales (N.E.E) puedan ser incorporadas 
URL: http://www.una.ac.cr/educare

CORREO: educare@una.cr

integralmente al plano social, con plenos derechos cívicos y ciudadanos, partiendo de la premisa fundamental de que son seres humanos con igualdad de derechos y deberes en el plano social, indistintamente de cuáles sean sus condiciones. Para ello, es preciso contar no solo con la respectiva conciencia al respecto, sino también con el manejo legal de esta dimensión, para proceder de acuerdo con lo que el sentido humanista y la ley rescatan.

Por otro lado, debe poseer un amplio y preciso conocimiento del marco legal relacionado con el sector educativo, esto implica el manejo circunstancial de diferentes aspectos de la vida ciudadana y legal de un país, no únicamente aspectos específicos, sino valorando de manera integral todo aquello que atañe a la vida social y sus repercusiones en los miembros de un país.

Inclusive, tal y como se puede apreciar, la formación de un profesional en educación es, sin lugar a dudas, amplia, precisa y compleja, a lo que se le debe agregar, pertinente. Asumir un grupo escolar no es una tarea fácil en la que prive la improvisación o la aplicación de estrategias sin una adecuada plataforma científica pedagógica. De este principio se parte para considerar que los profesionales en educación son científicos sociales y que su papel es quizás uno de los más importantes dentro de la sociedad, ya que procura, desde su dimensión ejecutiva, el desarrollo de habilidades y destrezas que le permitirán a los sujetos un desarrollo armonioso, tanto en el plano intrapersonal, como interpersonal, y los convertirán en agentes proactivos en el plano social.

Lo más importante y curioso de todo esto es que estas habilidades y destrezas, entre muchas otras que deben desarrollar, se alcanzan a lo largo de diversas aproximaciones en las realidades educativas, no basta, para ello, con un nivel tan importante y asimismo básico como lo es el diplomado universitario y mucho menos si se trata de condiciones prácticas en las cuales estas habilidades y destrezas se deben implementar de manera integral, amparadas, en la mayoría de los casos, desde escenarios totalmente vivenciales.

De acuerdo con todas estas características que va alcanzando desarrollar un estudiante universitario, se debe valorar el papel que juegan los profesores observadores, llamados también "profesores supervisores". Este último título es, quizás, el que mayor temor o preocupación les genera a los estudiantes observados, evaluados o medidos. Y es que si bien este tipo de acercamientos generan ciertos niveles de tensión en la población estudiantil universitaria, estos mismos no deben convertirse en momentos tortuosos o estresantes a tal grado que se lleguen a convertir en un mal recuerdo de su vida universitaria. Quizás un concepto amplio y pertinente que se podría utilizar es el de "profesor evaluador", este concepto no solo estaría direccionado a brindar una representación numérica de los logros del estudiante, sino que haría una valoración precisa de todas aquellas variables que pueden estar afectando de manera positiva o negativa su desempeño. Aquí se requiere, por lo tanto, de un perfil de profesor evaluador enriquecido en diferentes áreas, ya que no solo debe ser un profesional que determine aspectos didácticos o técnicos, sino que debe poseer el dominio de muchas otras habilidades, tales como las investigativas que le permitan posicionarse en los "zapatos" de cada estudiante, ya que no todos van a poseer las mismas construcciones y actitudes. Por ejemplo, en muchos casos se deben valorar aspectos como: ambiente en el cual se desempeñó el estudiante en sus años de 
formación escolar y colegial, características socio-económicas de su núcleo familiar y otros que puedan determinar con mayor aproximación sus características actitudinales.

Una profesora o profesor evaluador realizaría valoraciones acerca de si el estudiante presenta déficits formativos originados por un inadecuado tratamiento pedagógico desde los cursos universitarios que se brindan, aspecto que normalmente daría como resultado indagar el grado de efectividad que alcanza la formación universitaria. Este es un asunto complejo y delicado, ya que se podría llegar a la conclusión de que el estudiante presenta habilidades desarrolladas o carencias significativas como resultado de un inadecuado desarrollo formativo en los cursos que previamente a la intervención pedagógica ha llevado. Es aquí donde los profesores universitarios deben generar espacios reflexivos que les permitan constantemente evaluar su desempeño y garantizar, a través de los diferentes trabajos que los estudiantes desarrollan, su crecimiento intelectual y procedimental. Con esto no se quiere expresar que sea el profesor evaluador un juez de otros profesores, pero sí puede brindar resultados que les permitan a los profesores universitarios realizar conversatorios en los cuales se intensifique el análisis de lo que se percibe en la dimensión práctica.

Otro aspecto que podrían valorar los profesores evaluadores es el análisis de las características que posee tanto la institución educativa donde se ubica el estudiante universitario realizando su intervención pedagógica, como al mismo estudiante. Este punto es muy importante de rescatar y profundizar en selección, ya que cuanto mayor sea el liderazgo que presente la institución, mayores serán los espacios de acercamiento a diferentes dimensiones de formación integral. Idealmente, cada institución educativa se esfuerza por generar en la medida de lo posible momentos en los cuales los diferentes miembros de la comunidad se puedan involucrar y ampliar sus espacios educativos a través de actividades extracurriculares. Por ejemplo, una institución educativa que asuma su participación amplia en actividades extracurriculares como: banda escolar, grupos de bailes folklóricos, equipos deportivos, festivales de la creatividad, concursos de oratoria, concursos de poesía, cuento, dibujo, pintura, mimo, grupos teatrales, concursos de robótica y de recursos tecnológicos, y otros, sin lugar a dudas se convierte en un excelente modelo de creatividad para la formación de estudiantes que están comenzando a construir su futuro modelo profesional. Esto, por cuanto la riqueza de las experiencias aumentaría su percepción de lo que puede lograr un proceso educativo amplio y diverso. Claro está, una institución con estas características debe poseer un liderazgo evidente por parte de la dirección escolar, ya que previamente debe haber logrado motivar a los docentes, y a los padres y madres de familia para que apoyen y participen de estas actividades extracurriculares: ha logrado un equilibrio perfecto entre el currículum oficial y otras actividades extracurriculares. Tal y como lo ha indicado el Ministro de Educación, Leonardo Gardnier Rímulo, con la propuesta de Educación subversiva (TEDxPura VidaED, 2012).

Sería enriquecedor que el estudiantado pueda partir del análisis de diferentes instituciones y modalidades educativas, pues en algunos casos sus intervenciones se limitan a muy pocas instituciones a lo largo de toda su formación académica. Para ello, la naturaleza y las temáticas de los cursos universitarios podrían determinar si al estudiantado sería conveniente acercarlos 
URL: http://www.una.ac.cr/educare

CORREO: educare@una.cr

a escuelas unidocentes o multigrado; escuelas donde se brinde la modalidad de aula abierta (estudiantes con sobre edad); instituciones con énfasis científico, artístico, bilingüe, tecnológico, de educación diferenciada; con alfabetización de adultos; educación especial y cualquier otro tipo de oferta que enriquezca su conocimiento a partir de la confrontación de los diferentes contextos educativos a los cuales se les pueda aproximar. Ahora bien, parte de esta situación implicaría que los docentes universitarios asuman la elaboración de propuestas claramente definidas que les permitan a los estudiantes desarrollar habilidades y destrezas sustentadas en la observación crítica, analítica y reflexiva; que les permitan valorar las situaciones y condiciones desde una perspectiva propositiva.

En este punto, sería importante generar discusiones reflexivas acerca de la pertinencia de intervenir una o pocas instituciones con la participación de los profesores que imparten los cursos y, a partir de esto, generar intervenciones que le permitan a la institución educativa enriquecer su dinámica formativa; perfectamente se podría llegar, incluso, a procesos que inviten, en gran medida, a que las lecciones desde las aulas universitarias se trasladen directamente a las instituciones escolares. De ese modo, se podría intervenir de forma directa en situaciones tales como: manejo de grupo, adecuaciones curriculares, procesos metodológicos, análisis de la pertinencia de estrategias y materiales y, a partir de ello, la generación de nuevas propuestas, ambientación del aula, mejoramiento del rendimiento no solo académico, sino también el escolar y un sin número de valores agregados que podrían resultar de este tipo de intervenciones pedagógicas.

Además, es necesario valorar de manera sistemática algunos rasgos de la institución educativa, se pueden realizar apreciaciones objetivas sobre las características profesionales y actitudinales de la persona docente a cargo del grupo escolar y la dinámica pedagógica que desarrolla. Este punto es de gran relevancia, pues a partir de estas valoraciones se pueden reorientar algunas acciones que complementarán el proceso formativo universitario con mayor armonía. Valorar aspectos tales como: la relación que establece el estudiantado que realiza la intervención pedagógica con la persona docente a cargo del grupo escolar, el manejo de la disciplina que se establece a través los límites permitidos con el grupo escolar, el enfoque pedagógico que se utiliza, la experiencia profesional, la formación universitaria, la administración del currículo y otras características que pudiesen interferir con la intencionalidad planteada por parte del estudiantado universitario y que se pueda ver obstaculizado por cualesquiera de los puntos anteriores.

Al respecto, un ejemplo sencillo: Un estudiante universitario se encuentra ubicado en un tercer grado y el profesor evaluador le indica la necesidad de trabajar en ciertas asignaturas en sub-grupos; sin embargo, cuando el estudiante pone en práctica dicha sugerencia el caos impera en su intención, ya que el grupo ha venido trabajando desde primer grado en filas y en todas las asignaturas. En un caso hipotético como el anterior, no se le podría achacar al estudiante el fracaso de lo acontecido, ya que primero que nada sus intervenciones son esporádicas con el grupo y, quizás, ese tipo de estrategias requieran de una mayor intensidad, lo cual implica horas de práctica y, en segundo plano, el grupo desarrolla dinámicas a partir de 
lo que la docente titular implemente o no. Se genera, así, una cultura grupal que no se puede modificar de la noche a la mañana. Ahora bien, si un ejercicio como el anterior se realizara con la intención no solo de otorgar una calificación numérica, sino como un espacio de reflexión para que el estudiante proponga posibles causas de tal situación, la experiencia alcanzaría dimensiones distintas, es decir, en lugar de ser un espacio para resaltar el error reflejado en una calificación baja, se centraría en un análisis pedagógico profundo y significativo a partir de la práctica real para proponer, de manera natural, diferentes estrategias para solventar aquellos aspectos determinados como carentes. Tal y como se puede contemplar, el profesor evaluador debe ser hábilmente cuidadoso y certero en sus apreciaciones e intervenciones.

Asimismo, surge entonces la valoración que debe hacer el personal docente anfitrión, ya que no puede tomarse a la ligera su perfil. En círculos de discusión académica se proponen preguntas tales como: ¿Qué resulta más efectivo, la formación que se brinda desde las aulas universitarias o el modelo que ejerce el docente anfitrión con que se ubica el estudiante universitario? Interrogante de gran importancia, ya que el docente anfitrión se llega a convertir en un coformador de gran relevancia.

Quienes han estado a cargo de la orientación profesional del estudiantado universitario, incluido el autor de este artículo, sin lugar a dudas coinciden en que el perfil profesional del docente anfitrión termina siendo un pilar fundamental con su modelaje y, en muchos casos, inconscientemente el estudiante universitario termina ejecutando y reproduciendo prácticas sin percatarse. Algunas de estas, en ciertos casos, resultan poco pertinentes para la formación humana, pero, son funcionales para lograr implementar una disciplina amparada en la sumisión o el adoctrinamiento; fortalecen un ambiente en donde priva el temor hacia el adulto, el "orden" y hasta la excelencia académica del grupo escolar, la cual, erróneamente, podría estar sustentada en un reflejo ficticio de carácter numérico.

En relación con este último punto, no se quiere decir que las expresiones numéricas sean malas, quizás el punto preocupante estaría en los criterios que se utilizan para determinar dicha expresión numérica y, si en realidad, son un claro reflejo de una dinámica humanista, racionalista y constructivista. Estos tres pilares de la educación costarricense resultan vulnerables ante un ejercicio profesional desenfocado del principal interés de la educación: la autorrealización del individuo a partir de una educación integral amparada en valores. Por qué se puede afirmar que son vulnerables, bueno, porque en muchos casos el personal docente los pierde de vista y centra su atención en una expresión numérica que pasa a engrosar la papelería burocrática.

Esto mismo vendría a suceder con la calificación brindada al estudiantado universitario, ya que si bien se evalúan aspectos meramente conceptuales o teóricos, resulta de vital importancia valorar también lo actitudinal, esto es, su interés, disposición y actitud positiva ante los retos de su vida universitaria. El aspecto actitudinal en muchos casos podría encontrarse supeditado a otras dimensiones de carácter personal, familiar y social. Las vivencias emanadas de la intervención pedagógica deben convertirse en un manantial de información que le permita al cuerpo docente universitario conocer, con la mayor profundidad posible, todos aquellos 
URL: http://www.una.ac.cr/educare

CORREO: educare@una.cr

aspectos que pudiesen interferir en la construcción de aprendizajes por parte de cada uno de los estudiantes universitarios. Las sesiones de reunión en donde se valore el seguimiento que se les debe brindar, deben ser espacios para formular el trabajo en equipo, el apoyo individual y la aclaración de un panorama que les acerque de forma amplia y precisa a la labor profesional que van a desarrollar por varios años.

Como se ha venido analizando, la formación de las estudiantes y los estudiantes universitarios no es sencilla, ni pronta e inacabable, ya que requiere de la aplicación de múltiples estrategias educativas, cuyas principales características deben ser la adecuada direccionalidad entre sí y su integración, de tal manera que logren el fortalecimiento o desarrollo de habilidades que les permitan enfrentar, de la mejor forma todos aquellos retos que la labor educativa implica.

Si se parte de lo anterior, resulta fundamental valorar la pertinencia de que los estudiantes realicen, en su nivel de diplomado universitario, intervenciones profundas en las que asuman uno o varios grupos escolares. Quizás se podría pensar que no está del todo mal; sin embargo, los niveles de desarrollo profesional alcanzados en este nivel son básicos y escasos, sobre todo en cuanto a instrumental pedagógico y didáctico se refiere. Es ahí donde surge la pertinente necesidad de valorar si más bien las estudiantes y los estudiantes universitarios deberían enfrentar a los grupos escolares asumiendo una mediación directa o intervención pedagógica hasta que se encuentren concluyendo su nivel de bachillerato.

De acuerdo con la experiencia de quien escribe este artículo, es una situación importante de valorar, ya que los acercamientos con la población estudiantil se deben implementar y llevar a cabo desde las horas prácticas de cada curso universitario, tal y como se encuentra debidamente distribuido en la carga académica de los cursos. La formulación de un curso universitario implica el planificar qué tipo de trabajos y experiencias realizarán los estudiantes y las estudiantes y cómo eso se distribuirá entre los diferentes momentos educativos a los cuales se enfrente, tanto desde el desarrollo de las clases presenciales en las aulas universitarias como en la vivencia en las aulas escolares.

Es decir, todo profesor y profesora universitaria debe establecer diferentes estrategias prácticas en las que el estudiante confronte argumentos teóricos con la experiencia o realidad práctica; el aula universitaria se convertiría en un enriquecedor escenario en el cual se reconstruyan ese tipo de momentos; se dará paso a un diálogo permanente entre discursos a veces hipotéticos y las diferentes realidades existentes. Esto hace que el mismo profesor universitario valore cuán acertado está su discurso académico de acuerdo con lo que los estudiantes y las estudiantes expresan de sus resultados y estime si lo que podrían ser meramente "discursos ideales" son funcionales según lo que cada contexto puede demarcar.

Al respecto, en diversas ocasiones los estudiantes y las estudiantes han expresado que paradójicamente es más intensa la práctica de diplomado que la de bachillerato, situación contradictoria porque se podría estar afectando un adecuado tratamiento formativo dentro de la lógica epistémica de la formación universitaria, ya que a menor formación mayor rigurosidad o complejidad en los procesos experimentados. Ahora bien, no cabe duda alguna de que 
esto podría generar aprendizajes valiosos; el punto sería valorar a qué costo y si en realidad estas experiencias surgen de una intencionalidad educativa o son, sencillamente, azares del destino que ni siquiera son retomados, posteriormente, y que pueden quedarse como lagunas formativas. No es que se dude de la capacidad que tienen los estudiantes al respecto, ya que podrían reaccionar otorgando respuestas impresionantes y efectivas, aquí lo importante sería generar, de manera articulada y gradual, procesos armoniosos que enriquezcan intencional y positivamente el perfil de salida formativo que se desea alcanzar.

Todo curso universitario plantea objetivos correspondientes a intencionalidades y finalidades previamente coordinadas con lo establecido para cada nivel universitario. Debido a esto, es fundamental un análisis desde cada uno de estos niveles para determinar cuáles son las habilidades y destrezas cognoscitivas, socio-afectivas y psicomotrices que deben lograr los estudiantes a partir del tiempo de horas prácticas de cada curso. Quizás así se lograría, de forma sistémica, que la intervención pedagógica no se establezca como momento terminal de un nivel determinado, sino que se visualice como una cadena procedimental articulada que se iría construyendo eslabón por eslabón. Por eso mismo, se deber partir desde el primer curso y hasta el último que reciba la población estudiantil para visualizar la mayor efectividad y aprovechamiento de la formación brindada.

El personal docente universitario como profesional responsable del curso que imparte debe certificar lo que la población estudiantil ha logrado alcanzar; es decir, hacer mención tanto de las áreas fuertes como de los aspectos vitales por retomar en cada uno de sus estudiantes. Ahora bien, la valoración que se les realice a los estudiantes y las estudiantes al terminar un nivel académico debería estar, entonces, centrada en lo que el profesor del curso certifica, pero no debería servir únicamente para valorar el desempeño estudiantil, sino que también debe ser una fuente directa que contemple, tal y como se ha venido comentando, si en realidad el proceso como tal se encuentra debidamente articulado. Este ejercicio podría brindar información que ayude a determinar todos aquellos ajustes y enriquecería la proyección futura de los cursos.

Existe un criterio representativo y compartido, respaldado por comentarios de estudiantes de nivel de licenciatura, un sector representativo de docentes anfitrionas, profesores evaluadores e inclusive otros compañeros profesionales universitarios, respecto a que la intervención pedagógica que se realiza en diplomado debería trasladarse a un nivel de bachillerato, ya que la base formativa y la misma construcción académica sería más amplia y enriquecida.

Como se ha analizado, ubicar estudiantes en instituciones educativas para que realicen sus aproximaciones profesionales no se puede tomar a la ligera. No debe ser un proceso mecánico, debe ser considerada como la espina dorsal de la formación que se les brinda, además de convertirse con mayor fuerza en el gran reto de egresar profesionales con plataformas cognitivas eficientes que en realidad faciliten y promuevan, en el sistema educativo, mejores procesos de formación humana. La modalidad que se ofrezca, la intensidad, intencionalidad, pertinencia y gradualidad de estos procesos son aspectos indispensables a considerar, para satisfacer las 
URL: http://www.una.ac.cr/educare

CORREO: educare@una.cr

demandas de una sociedad compleja que es transformada a partir de la incorporación de nuevas y futuras generaciones.

Para finalizar y como un espacio de reflexión, se dejan planteadas las siguientes interrogantes: ¿Se puede garantizar que los procesos de acercamiento a la realidad educativa se encuentran debidamente organizados y enfocados hacia el mejoramiento del quehacer educativo?, ¿son en realidad procesos secuenciales articulados en un desarrollo gradual de habilidades y destrezas profesionales y personales?, ¿son las experiencias compartidas por los estudiantes universitarios un espacio para la reflexión académica y para la revalorización del discurso teórico?, ¿se ajustan los procesos evaluativos universitarios a la construcción cognitiva que han alcanzado los estudiantes de acuerdo con su vivencia y madurez académica?, ¿resultan ser estos procesos naturales, enriquecedores, diversos y bien orientados para una transformación positiva de la sociedad?, ¿es compatible el modelaje profesional de las docentes anfitrionas con la línea formativa que tiene la unidad académica?, ¿se abarcan inmediatamente desde la formación universitaria aquellos incidentes críticos que desestabilizan la intervención pedagógica?

\section{Referencias}

División de Educación Básica, Centro de Investigación y Docencia en Educación, Universidad Nacional. (s. f.). Curso de construcción pedagógica desde el aula escolar (DBJ-2012). Heredia, Costa Rica: Autor.

Ministerio de Educación Pública. (2005). Programas de Estudio del Ministerio de Educación Pública. San José, Costa Rica: Autor.

TEDxPura VidaED. (Productor). (2012). Educación subversiva [Video]. Recuperado de http://www. youtube.com/watch?v=C_ddnoHNbvQ

\section{Cómo citar este artículo en APA:}

Quesada, E. J. (2013). La intervención pedagógica como un reto de la formación universitaria: Hacia una práctica profesional articulada. Revista Electrónica Educare, 17(2), 167-182. Recuperado de http://www.revistas.una.ac.cr/index.php/EDUCARE/issue/current

Nota: Para citar este artículo en otros sistemas puede consultar el hipervínculo "Como citar el artículo" en la barra derecha de nuestro sitio web:

http://www.revistas.una.ac.cr/index.php/EDUCARE/index 